\title{
Emerging Trends on Abiotic Stress Tolerance Investigation in Crop Plants
}

\author{
Pandiyan Muthuramalingam ${ }^{1}$, Subramanian Radhesh Krishnan ${ }^{1}$, Subramani Pandian ${ }^{1}$ and Manikandan \\ Ramesh $^{1 *}$
}

${ }^{1}$ Department of Biotechnology, Science Campus, Alagappa University, India

Submission: August 29, 2017; Published: August 30, 2017

*Corresponding author: Manikandan Ramesh, Associate Professor, Department of Biotechnology, Science Campus, Alagappa University, Karaikudi, Tamil Nadu, India, Email: mrbiotech.alu@gmail.com

\begin{abstract}
In nature, plants are simultaneously exposed to number of biotic and abiotic stresses (BS and AbS), hence adversely affecting the overall plant growth and crop productivity. BS are easier to control unlike AbS are multigeneic and quantitative in nature. Thus, it is necessary to understand the molecular cross talks of environmental stress response and tolerance in plants. Therefore, for the improvement of crop plants, new methods and tools for genetic transformation is prerequisite. This could be addressed by ultrahigh-throughput computational biology. Especially, the advancement of constrains based omics approaches paves a way to characterize the plant cellular physiology under diverse AbS by publically available genomic, proteomic, ionomic, epigenomic, interactomic, metabolomic and phenomic data is used to increase agricultural crop productivity. Hence, is used to promote molecular based research in model plants as well as important crop plants. Further, the opinion will be useful for molecular biologist and plant physiologists to derive or design agronomically related strategies for the development of large spectrum AbS tolerant crops.
\end{abstract}

Keywords: Abiotic stress; Crop plants; Genomics; Proteomics; Metabolomics; Phenomics; Epigenomics; Interactomics; Ionomics

Abbreviations: BS: Biotic Stress; AbS: Abiotic Stress

\section{Introduction}

Emerging trends and enhancements in agricultural practices to speculate the global and future food security challenges are pivotal responses under changes in the climatic conditions. Now a days, crop yield is negatively encounter an increased number of biotic and abiotic stresses (BS and AbS), mainly on AbS [1-8]. AbS such as drought, salinity, submergence, metal, ozone, light, low and high temperatures are main destructive parameters on crop production. Precise measurement of plant morphomolecular traits particularly in field scale shows an essential role in the genetic trait improvement of crop plants. Therefore, computational studies are crucial to know about the molecular cross talks of AbS conditions on agricultural crop productivity.

Public availability of whole transcript sequences, recent game changing sequencing technologies have invigorated sequencing approaches in genomics and has developed the opportunities for more emerging analytical applications. Driven by technological advances, multiple new omics based research is powerful approach to understanding the molecular interactions and mechanism under AbS conditions. The advancement of omics and bioinformatics related approaches in genome, proteome, interactome, epigenome, hormonome, ionome and metabolome is a better platform to identify and understanding the molecular systematics that underlying the diverse plant functions especially the crop plants. Combined studies from omics related resources are specially focused on identifying the molecular system insights and biochemical properties that accelerates gene mining and its functional characters.

The era of genomics, proteomics, metabolomics and phenomics of crop stress biology involves transformation, mining and functional ontology annotation, promoter and SNP analysis, gene expression, pathway enrichment analysis, microRNA prediction, subcellular localization, gene structure analysis, physicochemical properties imputations, comparative analysis, interactome, protein function analysis, tissues specific and developmental stage expression analysis, simulation and focused on morpho-molecular differences in AbS exposed and affected crop plants and also the model plants give an novel insights on biological mechanisms that can hinder or regulate its survival. The identified potent key players into crop plants make an effort to generate $\mathrm{AbS}$ tolerance, enhancement of nutrients in crop plants. This omics based approaches are broadly used to 
understand the key players and their stress-specific traits that are needed to effectively target the different stress signalling pathways.

\section{Conclusion}

In conclusion, emerging advances in omics and bioinformatics approaches was to afford a well understanding about the abiotic stress $(\mathrm{AbS})$ responsible crop plant development. Moreover, in several crop plant species, the development of omics resources has been developed to focus the particular biological mechanisms of individual species. Incorporation of knowledge from omics based research is an advanced issue as plant molecular researchers seems to identify the importance, gaining molecular biological novel insights and promote translational research in AbS biology in crop plants.

\section{Acknowledgement}

PM acknowledges Alagappa University Research Fund (Ph.D./1215/AURF Fellowship/2015 dated 25.11.2015), Karaikudi, Tamil Nadu, India for providing Research Fellowship. The authors gratefully acknowledge the use of Bioinformatics Infrastructure Facility, Alagappa University funded by Department of Biotechnology, Ministry of Science and Technology, Government of India Grant (No.BT/BI/25/015/2012)..

\section{References}

1. Mittler R (2006) Abiotic stress, the field environment and stress combination. Trends Plant Sci 11(1): 15-19.
2. Prasad PVV, Pisipati SR, Momcilovic I, Ristic Z (2011) Independent and combined effects of high temperature and drought stress during grain filling on plant yield and chloroplast EF-Tu expression in spring wheat. J Agron Crop Sci 197(6): 430-441.

3. Atkinson NJ, Lilley CJ, Urwin PE (2013) Identification of genes involved in the response to simultaneouss biotic and abiotic stress. Plant Physiol 162(4): 2028-2041.

4. Narsai R, Wang C, Chen J, Wu J, Shou H, et al. (2013) Antagonistic, overlapping and distinct responses to biotic stress in rice (Oryza sativa) and interactions with abiotic stress. BMC Genomics 14: 93.

5. Prasch CM, Sonnewald U (2013) Simultaneous application of heat, drought, and virus to Arabidopsis plants reveals significant shifts in signaling networks. Plant Physiol 162(4): 1849-1866.

6. Pandey P, Ramegowda V, Senthil-Kumar M (2015) Shared and unique responses of plants to multiple individual stresses and stress combinations: physiological and molecular mechanisms. Front Plant Sci 6: 723

7. Ramegowda V, Senthil-Kumar M (2015) The interactive effects of simultaneous biotic and abiotic stresses on plants: mechanistic understanding from drought and pathogen combination. J Plant Physiol 176: 47-54.

8. Muthuramalingam P, Krishnan SR, Pothiraj R, Ramesh M (2017) Global transcriptome analysis of combined abiotic stress signaling genes unravels key players in Oryza sativa L.: An in silico approach. Front Plant Sci 8: 759. 